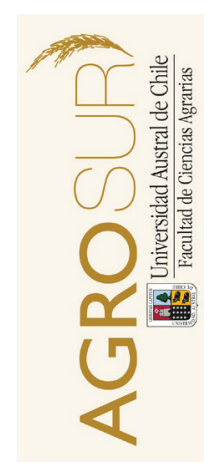

\title{
Inclusión del Nabo forrajero (Brassica rapa) como suplemento estival en dietas ofrecidas a vacas lecheras en predios de la Provincia de Ranco
}

\author{
Inclusion of summer turnip (Brassica rapa) as summer supplement \\ for dairy cows in farms of the Ranco Province
}

A R T I C LE INF O

Article history:

Received 22.10.2015

Accepted 15.03.2016

Keywords:

Summer Turnip

Grazing dairy cows

Milk Production

Multivariate analysis

Original Research Article,

Animal Science

${ }^{*}$ Corresponding author:

Sergio Aucal, Juan Pablo Keim

E-mail address:

juan.keim@uach.cl
Aucal, S. ${ }^{a}$, Balocchi, 0. ${ }^{b}$ Keim, J.P. ${ }^{b *}$

${ }^{a}$ Escuela de Agronomía Facultad de Ciencias Agrarias, Universidad Austral de Chile.

Instituto de Producción Animal, Facultad de Ciencias Agrarias, Universidad Austral de Chile. Casilla 567 Valdivia, Chile.

\section{RESUMEN}

El objetivo de este estudio fue analizar la oferta de alimentos, utilización del nabo forrajero y su relación con la producción de vacas lecheras en 35 predios de la Provincia del Ranco, Región de Los Ríos, Chile durante la época estival 2014. Se recopiló información de los rebaños lecheros, producciones lecheras, componentes de las dietas ofrecidas, manejo agronómico e inclusión del nabo forrajero.

La pradera constituyó la base de las dietas ofrecidas. La suplementación con nabo forrajero presentó un amplio rango de consumo, además se generalizó el uso de concentrados como suplemento ante una baja oferta de nabo forrajero y en menor cantidad con forrajes conservados y subproductos de la molinería.

El rendimiento del cultivo de nabo forrajero presentó un rango de 0,47 a 10,13 t MS/ha, variación influenciada por el manejo del cultivo y las condiciones edafo-climáticas de cada lugar. La inclusión relativa del nabo en la dieta presentó un rango de 2,6 a 40,7\% y la eficiencia de utilización promedió un 76,5\%.

El consumo total de materia seca y energía metabolizable fueron favorecidos al aumentar la oferta de nabo forrajero y concentrado comercial, presentando además una mayor producción lechera. Dietas con bajo consumo total de materia seca se caracterizaron por una baja producción lechera, independiente del nivel de inclusión de cada componente y la composición nutricional de la dieta.

Palabras clave: Nabo forrajero, vacas lecheras a pastoreo, producción de leche, análisis multivariado.

\section{INTRODUCCIÓN}

El principal recurso para la alimentación del ganado lechero bovino en las zonas templado húmedas como el sur de Chile son las praderas permanentes, las cuales destacan por su buena calidad y el bajo costo de producción en comparación a cualquier otro forraje destinado a la alimentación de vacas lecheras, lo que asegura la sustentabilidad y competitividad del negocio (Pulido et al., 2010). A pesar que el sur de Chile presenta características edafo-climáticas propicias para el desarrollo de especies pratenses, la variación de las condiciones climáticas presentes durante el año, limitan la tasa de crecimiento de las praderas a valores mínimos en meses desfavorables como los de invierno y de un verano seco (Keim et al., 2015). 
Las condiciones de verano seco o con baja pluviometría no sólo afectan la tasa de crecimiento de una pradera, sino que también la maduración fisiológica en las especies pratenses, afectando su calidad como alimento, reflejado por una caída del valor proteico y en su digestibilidad, mientras que el contenido de fibra aumenta lo que limita el consumo de materia seca y aporte de energía (Keim et al., 2015). Dependiendo del nivel de producción y carga animal bajo la cual se trabaje, el aporte de materia seca y nutrientes por parte de la pradera suele ser insuficiente para cubrir los requerimientos de las vacas en lactancia durante el verano, lo que resulta en una disminución en la producción de leche (Ruíz-Albarrán et al., 2015).

Ante este problema de déficit alimenticio, una alternativa efectiva para mantener la productividad es suplementar la dieta con otro componente distinto a la pradera permanente (García et al., 2008). Los alimentos suplementarios permiten aumentar el consumo de materia seca, suplir la deficiencia de uno ó más nutrientes, además de evitar el sobre pastoreo estival por falta de forraje. Entre las alternativas destacan los forrajes frescos, concentrados ó aditivos; siendo el nabo forrajero (Brassica rapa L.) uno de los principales cultivos suplementarios de uso estival (Lanuza, 2011).

En cuanto al rendimiento del Nabo forrajero, Parga et al. (2010) mencionan que se observan rendimientos de 10,14 t MS/ha, 12,28 t MS/ha y 13,63 t MS/ha para temporadas con pluviometría baja, normal y sobre lo normal, respectivamente.

El Nabo forrajero destaca por ser un cultivo simple de manejar en su establecimiento y pastoreo directo. Dentro de sus características nutricionales destaca su aporte energético, baja proteína, deficiencia en fibra efectiva, además de poseer limitaciones nutricionales a un cierto porcentaje de inclusión en la dieta diaria (Valentine y Kemp, 2007; Westwood y Mulcock, 2012).

La calidad nutricional del Nabo forrajero varía según la variedad, densidad de siembra, estado fenológico, fecha de siembra y la proporción hoja-raíz (Valentine y Kemp, 2007). Anrique et al. (2014) describen la calidad del Nabo forrajero para sus hojas con $14,2 \%$ MS; 21,56\% PC; 2,92 Mcal EM/kg MS y un 17,8\% FDN, mientras que para su raíz mencionan un 10,59\% MS; 15,62\% PC; 3,06 Mcal EM/kg MS y un 15,94\% FDN.

El Nabo forrajero se adapta a la capacidad de pastoreo del rebaño bovino, pero es necesario mantener un rígido control sobre la oferta diaria por su riesgo metabólico si es ofrecido en exceso y mantener la máxima eficiencia de consumo para evitar pérdidas ya sea por pisoteo, defecación o contaminación con tierra. Es por ello que se recomienda el uso de cerco eléctrico y respecto a la franja de oferta diaria para el rebaño debe ser larga y angosta (Barry, 2013).

La eficiencia de utilización es un punto clave del éxito de la suplementación, una eficiencia del $100 \%$ es señalada como óptima pero muy alejada de alcanzar. Diversos estudios reconocen un rango de variación de un $58 \%$ a $99 \%$ de eficiencia de utilización del cultivo (Barry, 2013). Por lo general las bajas eficiencias se dan en días de lluvia donde se forma lodo que contamina raíces y disminuye su preferencia en relación a las hojas que se encuentran sobre el suelo enlodado. A su vez Moate et al. (1999), señalan que bajo condiciones climáticas normales no existe un grado de preferencia por las hojas o por las raíces al momento del pastoreo.

Un exceso de Nabo forrajero en la dieta puede producir problemas a nivel metabólico como anemia hemolítica, bocio, fotosensibilización, lesiones hepáticas y timpanismo, por acción de los factores antrinutricionales como glucosinolatos, sulfóxido de metilcisteína y nitratos (Barry, 2013). Este consumo por animal no debería exceder el $30 \%$ de la dieta en base a materia seca consumida por día (Lanuza, 2011).

Respecto a la respuesta productiva, se ha observado incrementos de $0,6 \mathrm{~kg}$ de leche por $\mathrm{kg}$ MS de nabo suplementado en veranos secos, mientras que la respuesta ha sido marginal $(0,2 \mathrm{~kg}$ leche/kg MS nabo suplementado) en veranos húmedos (Moate et al., 1998; Parga et al., 2009, 2010). No obstante, la suplementación con nabo forrajero mejora el equilibrio en el aporte de energía y proteína para los microorganismos del rumen, al resultar en menores valores de urea en leche (Parga et al., 2010), mientras que complementar la suplementación de nabo forrajero con suplementos proteicos mejora la respuesta productiva (Moate et al., 1999).

Actualmente la utilización de Nabo forrajero es común en productores de leche bovina para suplementación estival (Anrique, 2011). No obstante, la información en cuanto a la utilización a nivel de campo de este cultivo suplementario y la relación de la inclusión de éste en la dieta con la producción de leche es limitada.

Considerando el interés que ha generado el cultivo de Nabo forrajero como suplemento en la dieta del ganado bovino lechero durante la época estival, el presente estudio tiene como objetivo general realizar un análisis sobre la utilización del Nabo forrajero y su relación con la producción lechera de 35 agricultores de la Provincia del Ranco, XIV Región de Los Ríos, Chile.

\section{MATERIAL Y MÉTODOS}

\section{Localización y duración del estudio}

El estudio se realizó en los meses de Enero y Febrero de 2014 en las comunas de La Unión (40 17' 00"; $73^{\circ} 05^{\prime} 00^{\prime \prime}$ ), Lago Ranco ( $\left.40^{\circ} 19^{\prime} 00^{\prime \prime} ; 72^{\circ} 30^{\prime} 00^{\prime \prime}\right)$ y

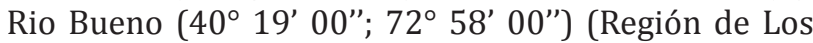
Ríos, Chile). Para ser incluidos en el estudio, los agricultores $(\mathrm{n}=35)$ visitados debían tener un período de post siembra del cultivo mínimo de 60 días y en pleno 
pastoreo de Nabo forrajero al momento de la visita; la distribución geográfica de los agricultores se muestra en la Figura 1. La duración del muestreo y recopilación de información se realizó durante enero y febrero del 2014, contemplando visitas, muestreos, procesamiento y secado de muestras.

\section{Información colectada}

Se utilizó una ficha técnica para recopilar información directamente con el productor referente al predio (Superficie del predio, número de vacas en producción, producción diaria) e información técnica referente al manejo general de los cultivos (variedad, dosis siembra, fecha siembra y cosecha) y a la producción lechera. Se determinó la producción promedio diaria de cada rebaño a partir de las variables litros diarios entregados a planta y el número de vacas en ordeña. Mediante un muestreo diario por predio se determinó la disponibilidad de pradera, nabo forrajero, y oferta de otros forrajes y alimentos concentrados.

El rendimiento del cultivo se determinó cosechando 4 marcos de $0,5 \mathrm{~m}^{2}$ en lugares representativos del cultivo, se pesó por separado la raíz de las hojas, obteniendo el peso fresco de ambos. De cada marco de cosecha se extrajo una submuestra, la cual fue pesada separando hojas de raíz, luego se refrigeró y transportó en un cooler hasta su procesamiento en laboratorio. El contenido de materia seca (\% MS) se determinó en base a las muestras obtenidas de cada predio, las cuales fueron laminadas y deshidratadas por separado (hoja y raíz) en un horno de aire forzado a $60^{\circ} \mathrm{C}$ durante 48 horas $(\% \mathrm{MS}=($ Peso seco*100) $/$ Peso fresco $)$ ).

La cantidad de nabo ofrecido (Kg MS/día) se determinó en base al rendimiento en materia seca, el área de la franja ofrecida diariamente y el número de animales consumiendo el cultivo. El residuo se determinó dentro de la franja de cultivo ofrecido el día anterior, para lo cual se pesó el material fresco (sin separar hojas de raíz) abarcado dentro de 4 marcos de $0,5 \mathrm{~m}^{2}$.

El consumo total diario del rebaño se determinó a partir de la diferencia entre la oferta diaria y residuo del día anterior, valor que posteriormente se dividió con el número total de animales que componían cada rebaño lechero.

La fitomasa de pradera disponible se estimó utilizando el plato medidor de altura comprimida mediante la fórmula: $Y=160 * x+250$, donde: $Y$ corresponde a la fitomasa de forraje (kg MS/ha) y $\mathbf{X}$ corresponde a la altura comprimida determinada con el plato, posteriormente el valor se corrigió en base a la superficie ofrecida diariamente, la cual fue medida mediante GPS.

El consumo de pradera se determinó por la diferencia obtenida entre la disponibilidad de forraje al momento de entrada y al momento de salida del potrero, valor que fue dividido entre el número total de animales del rebaño para obtener el consumo aparente individual diario.

En caso de existir suplementación adicional al nabo forrajero, al momento de la visita se determinó el tipo de suplemento (Concentrados comerciales, subproductos de la molinería, ensilaje, heno) y la cantidad ofrecida en base MS.

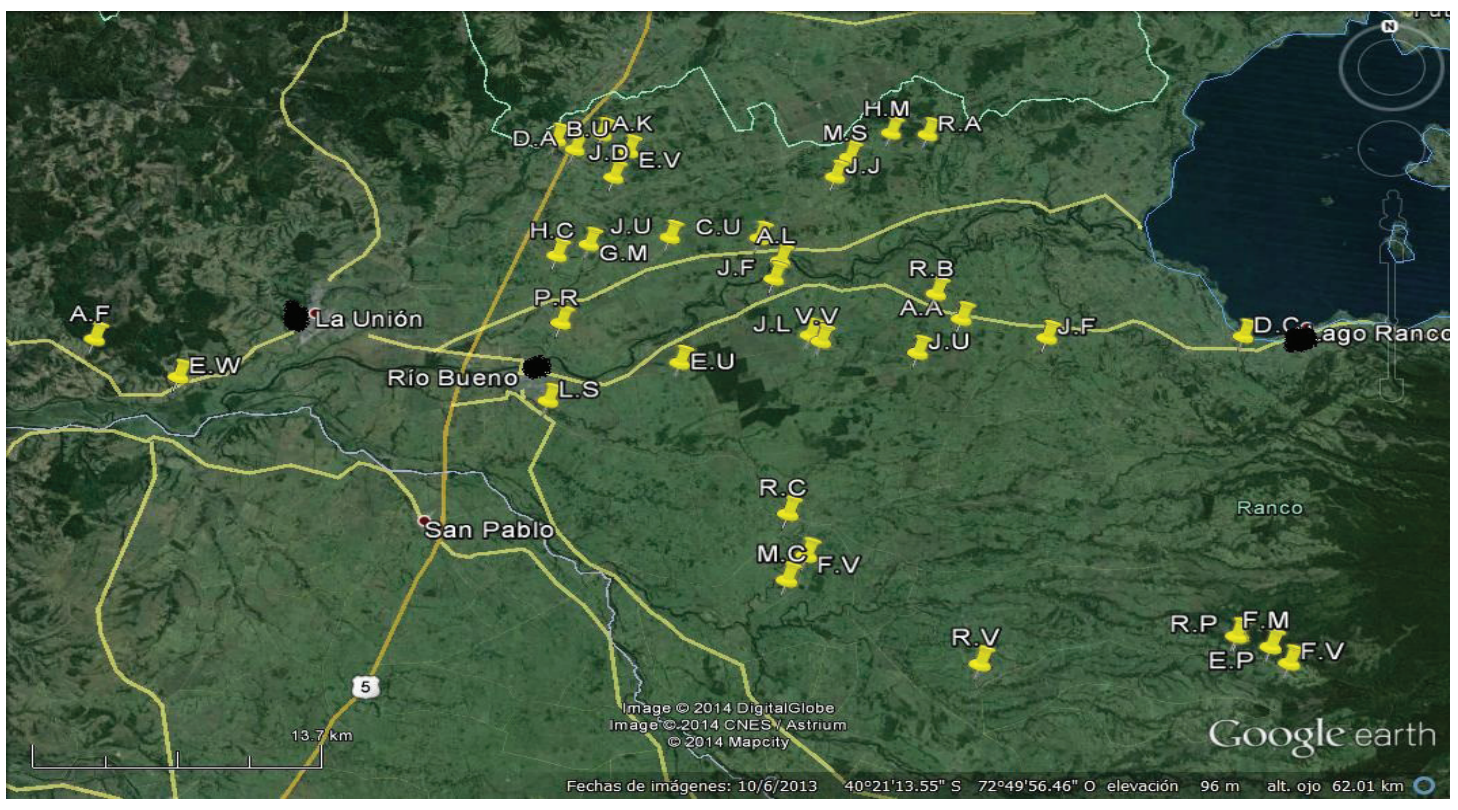

Figura 1. Ubicación de los predios visitados.

Figure 1. Localization of visited farms. 


\section{Estimación de la Composición nutricional de la dieta}

La concentración de proteína cruda (PC), fibra detergente neutro (FDN) y energía metabolizable (EM) del cultivo de nabo forrajero, pradera, forrajes conservados y subproductos de la molinería, se estimaron a partir de Anrique et al. (2014) y para el caso de los concentrados comerciales a partir de la composición nutricional declarada por el fabricante.

\section{Análisis estadístico}

Con el fin de caracterizar y describir las variables agronómicas del nabo forrajero y las dietas de los rebaños lecheros durante la época estival se utilizó estadística descriptiva con los datos obtenidos, determinando promedios, porcentajes relativos de participación, desviaciones estándar, valores mínimos y máximos, utilizando el programa EXCEL.

Para establecer las posibles relaciones de la dieta, su composición nutricional y la inclusión del Nabo forrajero con la producción de leche, se utilizaron técnicas de estadística multivariada, específicamente Análisis de Componentes Principales utilizando el software STATISTICA v. 7,0.

\section{RESULTADOS Y DISCUSIÓN}

\section{Antecedentes productivos prediales}

El tamaño promedio de los predios visitados fue de 31,4 $\pm 20,1$ ha (rango: 6,5 - 100) y contaban con un rebaño promedio de $35 \pm 22$ vacas (rango: 8 - 94). La producción lechera diaria por vaca de los rebaños estudiados varió dentro de un rango de 4,1 a 17,8 l y un promedio de 11,2 l, mientras que el consumo promedio total fue de 13,4 kg MS. En cuanto a la descripción de los rebaños, en todos los casos las razas fueron doble propósito (overo colorado y overo negro), con un peso promedio de $500 \mathrm{~kg}$ de peso vivo y se encontraban en el segundo tercio de lactancia.

\section{Antecedentes referentes al cultivo de Nabo forrajero}

La superficie destinada al cultivo de nabo forrajero fue de $2 \pm 1,5$ ha. En cuanto a la dosis de siembra utilizada, las cantidades se presentaron en un rango de 4 a $7 \mathrm{~kg} / \mathrm{ha}$. En todos los casos el método de siembra fue al voleo. Las dosis se encuentran sobre lo recomendado por De Ruiter (2009), quienes recomiendan 2 a $3 \mathrm{~kg} / \mathrm{ha}$ para siembra directa y $4 \mathrm{~kg} / \mathrm{h}$ a para siembra al voleo.

La fecha de siembra se distribuye desde la primera semana de octubre hasta la primera semana de diciembre, concentrándose un $74,2 \%$ de agricultores durante la segunda quincena de octubre y la primera quincena de noviembre. La fecha de siembra debe estar en función de la época estimada para ser utilizado el cultivo, la precocidad de la variedad y las características edafoclimáticas del lugar. Es por ello que la siembra debe realizarse entrada la primavera, una vez que el suelo alcanza la temperatura de $10^{\circ} \mathrm{C}$, siendo octubre el mes óptimo (White et al., 1999). También la fecha de madurez del cultivo para consumo, coincide con la baja en la tasa de crecimiento y de calidad de la pradera por la condiciones de verano no lluvioso (Balocchi, 2011).

En cuanto a la cosecha, ésta se inició durante enero por todos los agricultores, distribuídos uniformemente entre la primera y última semana de enero. El momento óptimo de cosecha es cuando las hojas alcanzan su máximo crecimiento y calidad nutricional con una altura referencial de $30 \mathrm{~cm}$ (Clark et al., 1996).

El Cuadro 1 presenta los valores mínimos y máximos, promedio y desviación estándar para rendimiento de materia verde, materia seca, porcentaje de materia seca, relación hoja/raíz y eficiencia de utilización del cultivo. Los rendimientos de materia seca del cultivo nabo forrajero, fluctuaron entre $0,47 \mathrm{t} \mathrm{MS} / \mathrm{ha}$ y 10,13 t MS/ha, con un promedio de 4,6 t MS/ha. Estos rendimientos se encuentran bajo el potencial señalado por White et al. (1999) de 11 t MS/ha, al ser sembrado en la primera quincena de octubre y un rendimiento promedio de $7 \mathrm{t}$ MS/ha para la misma variedad sembrada en la segunda quincena de octubre. Estudios a

Cuadro 1. Rendimiento, componentes del rendimiento y utilización del Nabo Forrajero.

Table 1. Yield, yield components and utilization efficiency of summer turnips.

\begin{tabular}{lccccc}
\hline & $\begin{array}{c}\text { Rendimiento kg } \\
\text { MV/ha }\end{array}$ & $\begin{array}{c}\text { \%MS planta } \\
\text { entera }\end{array}$ & $\begin{array}{c}\text { Rendimiento kg } \\
\text { MS/ha }\end{array}$ & $\begin{array}{c}\text { Relación } \\
\text { hoja/ raíz }\end{array}$ & $\begin{array}{c}\text { Eficiencia } \\
\text { utilización (\%) }\end{array}$ \\
\hline Máximo & 116.100 & 15,3 & $10.130,9$ & $1: 2,7$ & 94,9 \\
Mínimo & 4.700 & 8,1 & 471,3 & $1: 0,1$ & 27,1 \\
Promedio & $43.742,8$ & 11,3 & $4.681,9$ & $1: 1,1$ & 76,5 \\
d.e & $29.484,2$ & 2,1 & 2.610 & - & 14,9 \\
$\mathrm{n}$ & 35 & 35 & 35 & 35 & 35 \\
\hline
\end{tabular}


nivel nacional señalan rendimientos superiores al promedio de este estudio con 10,14 t MS/ha en temporada seca, 12,28 t MS/ha en temporada normal y 13,63 t MS/ha para una temporada lluviosa (Torres, 2013). El rendimiento puede variar ampliamente en función de la pluviometría de la estación estival (Lanuza, 2011). $\mathrm{Si}$ bien algunos agricultores obtuvieron rendimientos cercanos a los ensayos citados, la amplia variación está influenciada por el manejo (la mayoría no recordó dosis de fertilización ni realizó control de malezas) y el tipo de suelo sobre el cual se estableció el cultivo. Los bajos rendimientos están asociados al predios del sector Trumao-La Unión con siembras tardías, que en relación a Río Bueno la precipitación acumulada en los meses de diciembre de 2013 a Febrero de 2014 fue de $50 \mathrm{~mm}$ menos (INIA, 2016) y se encontraban en suelos de la serie La Unión, de tipo rojo arcilloso los cuales tienen menor productividad que los suelos "trumaos" del Llano Longitudinal, como consecuencia de un mayor déficit hídrico asociado a la lenta permeabilidad producto del componente arcilloso y menor contenido de materia orgánica (Bernier, 1982).

La materia seca promedió un $11,3 \%$ como planta entera, valor cercano a lo reportado por Anrique et al. (2014) de 11,41\% MS como planta entera y Moate et al. (1998) que registraron 8-10\% MS, indicando al Nabo forrajero como un alimento con baja cantidad de materia seca, pero muy digestible $(88,2-93,2 \%)$.

La relación hoja/raíz presentó un promedio de 1:1,1. Lanuza (2011), destaca la importancia de la pluviometría sobre la relación hoja-raíz, que puede variar de 2:1 a principios del verano hasta relaciones inversas de 1:2 a fines de la estación o un verano muy seco. White et al. (1999) y De Ruiter et al. (2009) señalan que la relación hoja-raíz depende del grado de desarrollo fenológico del cultivo, la variedad y las condiciones ambientales, proponiendo como momento óptimo de cosecha cuando un $30-50 \%$ del peso total de la planta corresponde a la raíz. El valor promedio obtenido del estudio se acerca a lo descrito por estos autores.

La eficiencia de utilización del cultivo fluctuó entre un $27,1 \%$ y un $94,9 \%$, con un promedio de $76,48 \%$, promedio bajo lo registrado por Moate et al. (1999), quienes reportan una eficiencia de utilización con un promedio del $90 \%$ y a su vez destacan que una baja eficiencia de utilización se da en días de lluvia donde las raíces quedan pisoteadas y con lodo. De Ruiter et al. (2009) señalan que el pastoreo debe realizarse utilizando cerco eléctrico en franjas con un frente amplio $\mathrm{y}$ angostas con el objetivo de disminuir las pérdidas y aumentar la eficiencia de utilización. La mayor parte de los agricultores mantuvo un alto porcentaje de utilización, los menores valores registrados se asocian al mal cálculo de franja de oferta del cultivo, derivando en un pisoteo de hojas y raíz, provocando así rechazo al momento del pastoreo.

\section{Caracterización dietas ofrecidas a los rebaños lecheros.}

Las dietas ofrecidas a las vacas en cuanto a sus componentes (tipo), consumo (kg), participación relativa (\%) y aporte nutricional (FDN, EM y PC) se presentan en los Cuadros 2 y 3 . Se observa una variación en cuanto a los componentes de la dieta y la cantidad ofrecida de cada alimento por los 35 agricultores participantes del estudio. El consumo diario de Nabo forrajero por vaca fluctuó en un rango de 0,28 a 7,06 kg MS y un promedio de 2,8 kg MS, mientras que la participación relativa promedio en la dieta fue del 20,2\% (Cuadro 2). De Ruiter et al. (2009) mencionan que el consumo diario no debe ser mayor a $5 \mathrm{~kg} \mathrm{MS} /$ vaca y que una parti-

Cuadro 2. Estadística descriptiva para componentes de las dietas ofrecidas a vacas lecheras en pastoreo estival.

Table 2. Descriptive Statistics for the diets offered to grazing dairy cows in summer.

\begin{tabular}{|c|c|c|c|c|c|c|c|}
\hline & Nabo & Pradera & Concentrado & Heno & Ensilaje & Molienda & Total \\
\hline \multicolumn{8}{|c|}{ Cantidad de Alimento Ofrecido (kg MS/día) } \\
\hline Máximo & 7,1 & 14,6 & 3,5 & 6,7 & 2,4 & 1,8 & 19,3 \\
\hline Mínimo & 0,3 & 3,5 & 0,0 & 0,0 & 0,0 & 0,0 & 5,8 \\
\hline Promedio & 2,8 & 9,6 & 0,7 & 0,4 & 0,1 & 0,2 & 13,8 \\
\hline d.e & 1,8 & 3,03 & 0,9 & 1,4 & 0,5 & 0,5 & 3,6 \\
\hline \multicolumn{8}{|c|}{ Proporción de Pradera en la Dieta (\%) } \\
\hline Máximo & 40,7 & 97,4 & 19,6 & 51,4 & 18,6 & 11,5 & - \\
\hline Mínimo & 2,6 & 26,4 & 0,0 & 0,0 & 0,0 & 0,0 & - \\
\hline Promedio & 20,2 & 69,4 & 5,1 & 3,1 & 1,1 & 1,1 & - \\
\hline d.e & 10,6 & 14,0 & 6,6 & 12 & 3,2 & 3,2 & - \\
\hline
\end{tabular}


Cuadro 3. Concentración y consumo de nutrientes (FDN, EM y PC) en vacas lecheras a pastoreo en verano.

Table 3. Nutrient (NDF, ME and CP) concentration in the diet and intake by grazing dairy cows in summer.

\begin{tabular}{lcccccc}
\hline & \multicolumn{2}{c}{ FDN } & \multicolumn{2}{c}{ EM } & \multicolumn{2}{c}{ PC } \\
\hline & (kg/vaca/día) & $\mathbf{( \% )}$ & (Mcal/ vaca/día) & (Mcal/kg MS) & (kg/vaca/día) & (\%) \\
\hline Máximo & 8,3 & 51,2 & 52,2 & 2,7 & 3,3 & 18,6 \\
\hline Mínimo & 2,5 & 35,3 & 14,9 & 2,0 & 1,1 & 9,4 \\
Promedio & 5,9 & 42,8 & 34,4 & 2,5 & 1,9 & 13,9 \\
d.e & 1,5 & 4,1 & 9,4 & 0,2 & 0,7 & 3,1 \\
\hline $\mathrm{n}$ & 35 & 35 & 35 & 35 & 35 & 35 \\
\hline
\end{tabular}

cipación relativa del Nabo forrajero en la dieta sobre $30 \%$ puede afectar la salud animal por la presencia de factores anti nutricionales (Moate et al., 1999; Lanuza, 2011). La mayor parte de los agricultores mantuvo los niveles de inclusión de Nabo forrajero dentro de los límites recomendados, evitando así la presencia de problemas metabólicos y características organolépticas de este alimento en la leche.

El consumo diario de pradera por vaca fluctúo en un rango de 3,45 a 14,64 kg MS y posee una participación relativa promedio en la dieta de 69,4\% (Cuadro 2). La variación en la oferta diaria de pradera, dependió de la fitomasa y la carga animal, lo que está determinado por factores como el manejo y las características edafoclimáticas. Keim et al. (2015) describen una disminución progresiva de la disponibilidad y valor nutritivo de la pradera durante el verano (disminuye el contenido proteico y energético, aumenta la participación de carbohidratos estructurales), restringiendo el consumo de MS. Dillon (2006), indica a la pradera con buen manejo como el alimento de menor costo en cuanto a la inversión anual, es por ello que a medida que aumenta su inclusión en la dieta, aumenta el margen económico para el productor. Por lo tanto, los agricultores que poseen un menor grado de inclusión de pradera por baja disponibilidad, se ven obligados a invertir en alimentos de mayor costo para suplir el déficit de oferta diaria de forraje, con el objetivo de evitar una baja considerable de la producción láctea.

El consumo promedio diario de concentrado por vaca fue de 0,7 kg MS y una participación promedio en la dieta cercana al 5,1\% (Cuadro 2). Keim y Anrique (2011), remarcan el mayor costo por kg MS de concentrado en relación al costo por kg MS de forraje pratense, por ello recomiendan el uso estratégico de concentrados, considerando la respuesta a la suplementación, el precio pagado por litro de leche y el costo del concentrado.

En cuanto al uso de forrajes conservados y sub productos industriales, son pocos los agricultores que utilizan estos alimentos como suplemento estival. Se observó un consumo diario promedio por vaca de 4,27 kg MS de heno, 1,74 kg MS de ensilaje y 1,24 kg MS de afrecho de trigo y una participación relativa promedio en la dieta de $5,1 \%, 3,1 \%$ y $1,1 \%$ (Cuadro 2) siguiendo el mismo orden anterior. Anrique (2013), destaca el uso de forrajes conservados como heno y ensilaje como alternativa válida frente a la escasez de forraje pratense por las condiciones de verano, su menor costo que un concentrado comercial y el resguardo para evitar el sobrepastoreo bajo las condiciones de baja tasa de crecimiento de la pradera (Lanuza, 2011). El ensilaje mejora las condiciones ruminales cuando el corte se realiza en el momento óptimo y posee una mejor calidad nutricional que la pradera espigada de verano (Anrique, 2013), el heno por su parte aporta fibra efectiva pero muy poco de proteína y energía metabolizable a la dieta (Lanuza, 2011). La inclusión de forrajes conservados en los predios estudiados tuvo como objetivo evitar una baja considerable en la producción lechera, dada la baja tasa de crecimiento pratense y la precaución señalada por los agricultores de acompañar con un forraje tosco la inclusión de nabo forrajero en la dieta. Por otra parte, la sustitución de pradera por forraje conservado ayuda a evitar el sobrepastoreo estival. En el Cuadro 2 se observa el rango de consumo diario total de alimentos por vaca que fue de 5,79 a 19,34 kg MS, y un promedio de 13,8 kg MS. Según la metodología de cálculo de consumo de MS propuesta por Anrique et al. (2014), en función del segundo tercio de lactancia, el peso promedio estimado del rebaño y los valores mínimo, máximo y promedio de producción de leche diaria, el consumo óptimo de materia seca, debería encontrarse en un promedio de $15 \mathrm{~kg}$ MS. Niveles de consumo total de materia seca por debajo del rango recomendado explicarían en gran parte los bajos niveles de producción lechera observados para algunos casos.

El Cuadro 3 reporta la concentración y cantidad de FDN, EM y PC de las dietas ofrecidas durante la época estival. El consumo de FDN presentó un rango de participación relativa en la dieta que va de $35,29 \%$ a $51,23 \%$, y un promedio de $42,83 \%$. Kolver y de Veth (2002) mencionan la importancia de la fibra dada su 
composición estructural (celulosa, hemicelulosa y lignina) que está relacionada con una fermentación lenta y menor producción de ácidos en el rumen, lo que ayuda a estabilizar el pH. Por su parte la fibra efectiva está relacionada con el tamaño de la partícula de FDN, influyendo sobre la masticación, salivación y naturaleza bifásica del contenido ruminal. Los mismos autores recomiendan para una dieta basada en pradera incluyendo suplementación con alimentos de alto contenido de almidón, un rango de 27 a 33\% de FDN en la dieta y 20\% de FDN. El máximo coincide con el mínimo registrado en este estudio, hay un porcentaje de agricultores que ofrece las óptimas condiciones de alimentación requeridas para un buen funcionamiento ruminal, mientras que los valores más altos registrados podrían afectar el consumo de MS, producto de una menor y más lenta degradación de los alimentos a nivel ruminal (Zebeli et al., 2012). No se realizó una estimación del consumo de fibra físicamente efectiva, pero dada las condiciones de madurez fisiológica pratense, aporte de heno y ensilaje, no debería encontrarse bajo las cantidades recomendadas (Holmes et al., 2002).

La Energía Metabolizable consumida diariamente por vaca se presentó en un rango de 14,95 a 52,15 Mcal de EM y en cuanto a la Energía Metabolizable presente en las diferentes dietas ofrecidas, presentó un promedio de 2,5 Mcal EM/kg MS. Kolver (2003), señala que el consumo de energía es el principal factor limitante de producción en vacas bajo condiciones de pastoreo y en cuanto a la cantidad de energía requerida afirma que está en función del metabolismo basal, la actividad diaria y la producción lechera. Keim y Anrique (2011), señalan la importancia de considerar los requerimientos energéticos de los microorganismos del rumen, que aumentan su población y son fuente de aminoácidos al pasar al intestino. Según la metodología de cálculo propuesta por Anrique et al. (2014) basada en la información productiva del rebaño, el consumo diario de energía metabolizable por vaca debería encontrarse alrededor de 35,33 Mcal de EM. Los mayores consumos de energía coinciden con mayores producciones de leche, mientras que los menores aportes energéticos tienden a limitar la producción, por ende los planteles producen muy por debajo de su potencial.

La concentración promedio de fue de 13,9\% BMS. Según la metodología de cálculo propuesta por Anrique et al. (2014), y basada en la información productiva del rebaño, la participación relativa de la proteína cruda debería ser del $14-16 \%$ respecto al consumo total de materia seca diario por vaca. Jacobs y Hargreaves (2002), mencionan que respecto al metabolismo proteico es importante considerar tanto las necesidades de la vaca como de la flora microbiana del rumen, ya que los microorganismos que pasan al intestino delgado, son degradados por las enzimas digestivas, aportando aminoácidos al rumiante. La proteína mi- crobiana es capaz de mantener una producción lechera de hasta 12 l diarios por vaca, una vez superado este volumen es necesario aportar proteína que escape de la fermentación ruminal o el consumo microbiano, pasando directo al intestino. En algunos casos, los valores registrados se encuentran bajo lo recomendado, lo que se explicaría en la disminución en la concentración de PC de la pradera y la baja PC característica para el nabo forrajero y suplementos energéticos (Anrique, 2013). En ningún caso existió una suplementación proteica, situación que permitiría aumentar la respuesta productiva según lo observado por Moate et al. (2002) en vacas a pastoreo estival que consumen nabo forrajero.

\section{Relación entre producción lechera y dieta ofrecida.}

La Figura 2 presenta las variables incluidas (PL: Producción de Leche; Cnab: Consumo de nabo; Cpr: Consumo de pradera; Ccon: Consumo de concentrado; CMSt: Consumo de materia seca total; FDNc: Consumo de fibra detergente neutra; EMc: Consumo de energía metabolizabe; PCc: Consumo de proteína cruda: \%nab: Participación relativa de nabo en la dieta; \%prad: Participación relativa de pradera en la dieta; \%con: Participación relativa del concentrado en la dieta; \%FDN: Participación relativa de la fibra detergente neutra en la dieta; \%PC: Participación relativa de la proteína cruda en la dieta) en el análisis de componentes principales y su respectiva ubicación relativa en los componentes principales (CP) 1 y 2 , que en conjunto explican el $71,16 \%$ de la variabilidad observada en el estudio. El CP 1 explica el $42,85 \%$ de la variación total, mientras que el CP 2 por su parte explica un $28,31 \%$ de la variabilidad total y las variables de mayor relevancia absoluta son Cnab con signo positivo y Ccon con signo negativo. En la Figura 3, se presenta la distribución de los agricultores considerados en el estudio en función de las características de la dieta ofrecida y su producción láctea. Las características de consumo y producción asignadas a cada cuadrante y su relación con los datos reales de la dieta y producción, determinaron la ubicación de cada agricultor en el gráfico de dispersión, información que se presenta y analiza en la Figura 3.

$\mathrm{Al}$ analizar las proyecciones de los vectores de relevancia y su correlación con los ejes en la Figura 2, para el CP 1 , se observa una relación positiva entre CMSt, Cnab, Ccon, Cpr y PL, esto indica que los agricultores que ofrecieron mayor cantidad de alimento, obtuvieron mayor producción lechera. Por último, tiene importancia la relación positiva entre Cnab y Ccon con EMc, esto significa que las dietas con mayor oferta de nabo forrajero y concentrado, presentaron mayor aporte energético.

Respecto al CP 2, las variables Cnab y Ccon tienen una relación inversa, es decir agricultores con alta oferta de Nabo forrajero, ofrecen poco o nula cantidad 


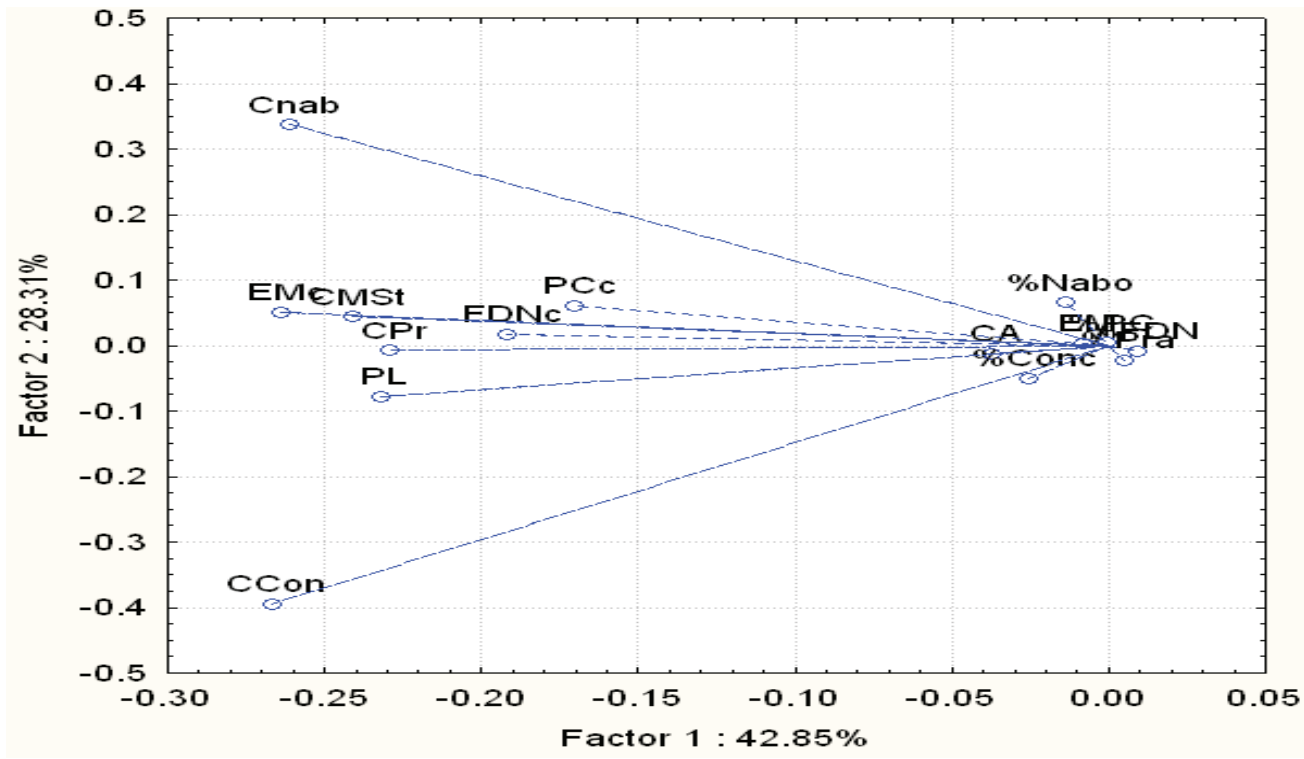

Figura 2. Distribución de las variables evaluadas en los Componentes Principales 1 y 2.

Cnab: consumo de nabo; EMc: consumo de energía metabolizable; CMSt: consumo de materia seca total; CPr: consumo de pradera; PL: Producción de leche; CCon: consumo de concentrado; PCc: PC consumida; FDNc: FDN consumida; \%Nabo: proporción relativa de nabo en la dieta; CA: carga animal; \%Conc: proporción relativa de concentrado en la dieta; \%Pr: proporción relativa de pradera en la dieta; FDN: concentración de FDN en la dieta; PC: concentración de PC en la dieta; EM: concentración de EM en la dieta.

Figure 2. Distribution of analized variables in Principal Components 1 and 2.

Cnab: turnip intake; EMc: metabolizable energy intake; CMSt: dry matter intake; CPr: pasture intake; PL: milk yield; CCon: concentrate intake; PCc: CP intake; FDNc: NDF intake; \%Nabo: turnip proportion in the diet; CA: stocking rate; \%Conc: concentrate proportion in the diet; \%Pr: pasture proportion in the diet; FDN: dietary NDF concentration; PC: dietary CP concentration; EM: dietary ME concentration.

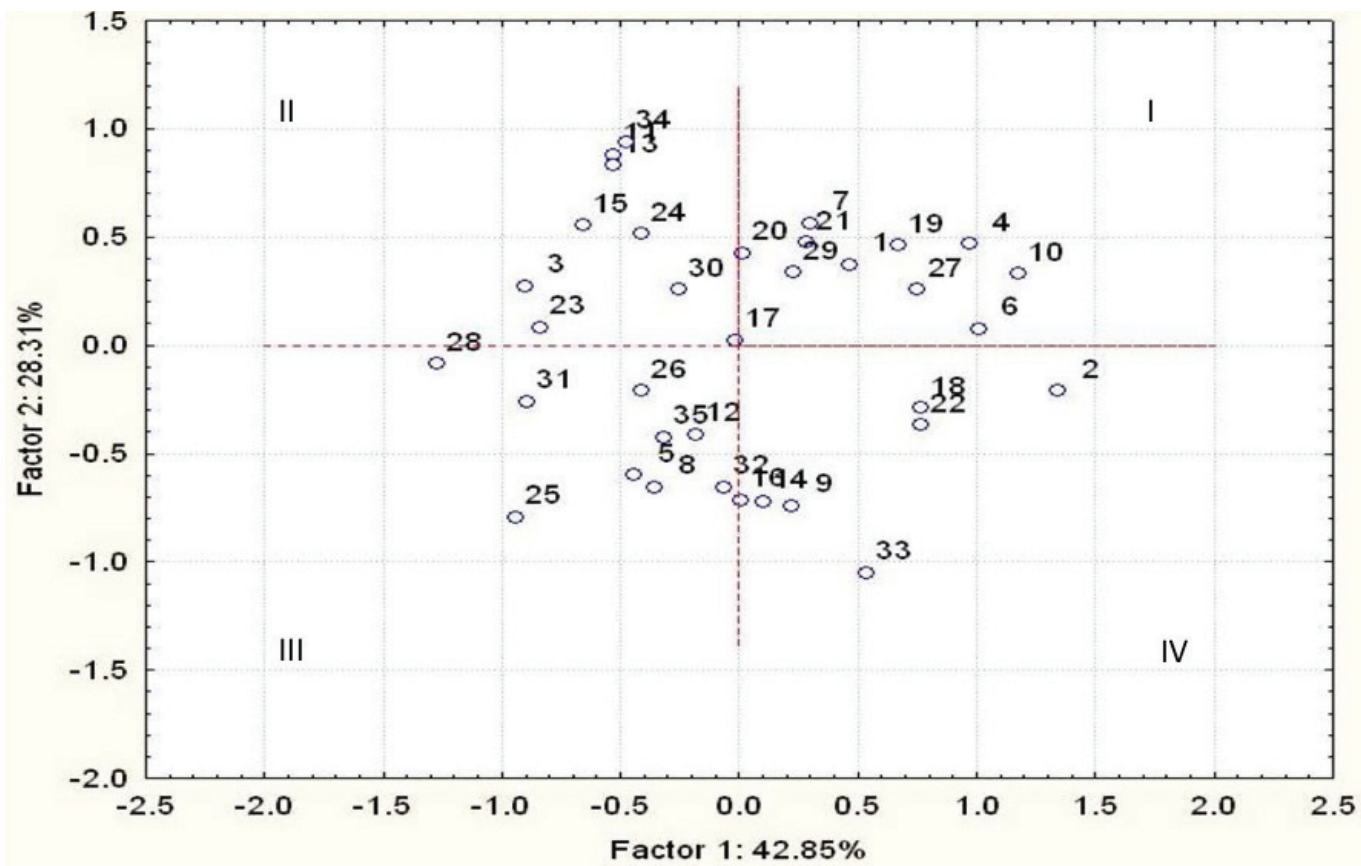

Figura 3. Distribución de los predios estudiados en relación a los Componentes Principales 1 y 2.

Figure 3. Distribution of the studied dairy farms according to Principal Components 1 and 2. 
de concentrado y agricultores con baja oferta de Nabo forrajero suplen con alta oferta de concentrado energético. Es decir, los agricultores estarían utilizando el nabo forrajero en reemplazo del concentrado. Ambos alimentos tienen la característica de aportar altas cantidades de energía metabolizable, mediana cantidad de proteína y bajo aporte de fibra, por tanto la sustitución de uno por otro no implica una gran variación de aporte nutricional (EM, FDN y PC).

El cuadrante 1 (Figura 3) agrupa el 28,5\% de los agricultores, los cuales presentaron una oferta diaria de alimentos bajo la media, caracterizada por una nula ó baja participación relativa de concentrados, alta participación relativa de nabo forrajero y una producción lechera bajo la media. El cuadrante II agrupa el 25,7\% de los agricultores, los cuales presentaron una oferta diaria de alimentos sobre la media, caracterizada por una nula ó baja participación relativa de concentrados, alta participación relativa de nabo forrajero y una producción lechera sobre la media. El cuadrante III agrupa $25,7 \%$ de los agricultores, los cuales presentaron una oferta diaria de alimentos sobre la media, caracterizada por una alta participación relativa de concentrados, baja participación relativa de nabo forrajero y una producción lechera sobre la media. Por último el cuadrante IV agrupa el $20 \%$ de los agricultores, los cuales presentaron una oferta diaria de alimentos bajo la media, caracterizada por una alta participación relativa de concentrados, baja participación relativa de nabo forrajero y una producción lechera bajo la media.

Clark et al. (1997), realizaron un estudio con vacas durante el segundo tercio de lactancia con una dieta basada en pradera suplementada con 4 a $8 \mathrm{~kg}$ MS de nabo forrajero. Concluyeron que a medida que aumentaba el nivel de suplementación con nabo forrajero, aumentaba el consumo total de MS, lo cual aumenta la producción lechera del rebaño. Moate et al. (1998), por su parte suplementaron con 3, 4 y $6 \mathrm{~kg}$ MS de nabo forrajero lo que correspondió a una participación relativa de $19-25-38 \%$ en la dieta basada en ensilaje de pradera y heno, los resultados obtenidos indicaron una producción de 16,1; 16,3 y 14,9l leche por vaca diariamente, concluyendo que la inclusión del nabo forrajero puede ayudar mantener una alta producción lechera, pero es necesario suplir el déficit proteico en la dieta. Parga et al. (2009), han evaluado la suplementación estival con nabo forrajero y concentrado comercial en rebaños lecheros a mediados de lactancia, observando una mayor respuesta productiva a la suplementación con concentrado $\mathrm{v} / \mathrm{s}$ nabo forrajero $(0,78 \mathrm{v} / \mathrm{s} 0,5 \mathrm{~kg}$ de leche/kg suplemento), no obstante la suplementación con nabo forrajero puede resultar en un mejor retorno económico ya que el costo por kg MS es de alrededor de un tercio del costo por kg de concentrado (Eckard et al., 2001). La respuesta a la suplementación con nabo forrajero disminuye a $0,2 \mathrm{~kg}$ de leche $/ \mathrm{kg}$ suplemento en veranos húmedos con mayor disponibilidad de forraje. Los resultados obtenidos del estudio coinciden con los obtenidos por los autores citados a nivel nacional e internacional, respecto al efecto positivo del aumento de la oferta de alimentos sobre la producción lechera.

Una alta participación relativa de nabo forrajero o concentrado comercial no asegura una mediana ni alta producción lechera, cuando se presentan bajos aporte de pradera y baja oferta total de materia seca en una dieta, caso presentado por el $48,6 \%$ de los agricultores estudiados, siendo el consumo de energía y materia seca el factor más relevante para la producción de leche. Es por ello que al aumentar el consumo de alimentos se ve favorecida la producción lechera, caso presentado por el $51,4 \%$ de los agricultores estudiados, que además de utilizar suplemento nabo o concentrado tienen en general buen manejo sobre la pradera, lo que les permitió rezagar y ofertar este forraje durante la época estival.

Finalmente, el aumento energético en la dieta ocurrió en función del aumento del consumo de alimentos energéticos como el nabo forrajero y el concentrado comercial, dada la naturaleza de sus componentes, como son los carbohidratos no estructurales (almidón y otros azúcares).

\section{CONCLUSIONES}

Se observó una amplia variación en el rendimiento del nabo forrajero. Casos de bajos rendimientos se asocian a siembras tardías y zonas agroclimáticas de menores precipitaciones.

Respecto a la inclusión de nabo forrajero, este fue utilizado en diferentes proporciones en la dieta. La proporción de nabo en la dieta no se relacionó con la producción láctea, siendo el consumo de MS y energía los factores que mejor explicaron la respuesta productiva. En general el nabo forrajero se utilizó como alternativa a la inclusión de concentrados comerciales.

\section{REFERENCIAS}

Anrique, R., 2011 Análisis de Características del Consumo de Nueve Predios Bajo Monitoreo en dos Escenarios Pluviométricos. Plan de Desarrollo Lechero Watt's. Publicación $\mathrm{N}^{\circ} 1$, Año 2. Osorno, Chile.

Anrique, R., 2013. Nutrición y Alimentación de Vacas Lecheras en Pastoreo. CORFO. Osorno, Chile.

Anrique, R., Molina, X., Alfaro, M., Saldaña, R., 2014. Composición de alimentos para el ganado bovino. Fundación para la Innovación Agraria, FIA, Ministerio de Agricultura, MINAGRI. Universidad Austral de Chile. Valdivia, Chile.

Balocchi, O., 2011. La Pradera Como Alimento, in: Pulido, R., Parga, J., Lanuza, F. Balocchi, O. (Eds.), Suplementación de vacas lecheras a pastoreo. Consorcio Tecnológico de la Leche S.A. Osorno, Chile. pp. 11-20 
Barry, T.N., 2013. The feeding value of forage brassica plants for grazing ruminant livestock. Animal Feed Science and Technology 181, 15-25.

Bernier, R., 1982. Fertilización de Praderas. I. Suelos de la X Región. Boletín Técnico № 57. Instituto de Investigaciones Agropecuarias (INIA). Osorno, Chile, pp. 1 - 12

Clark, D.A., Howse, S.W., Johnson, R.J., Pearson, A., Penno, J.W., Thomson, N.A., 1996. Turnips for summer milk production. Proceedings of the New Zealand Grassland Association 57, 145-50.

Clark, D. Harris, S. Thom, E. Waugh, C. Copeman, P., Napper, A., 1997. A comparison of Barkant turnips and Superchow sorghum for summer milk production. Proceedings of the New Zealand Grassland Association 59, 157-161.

De Ruiter, J., Wilson, D., Maley, S., Fletcher, A., Fraser, T., Scott, W., Berryman, S., Dumbleton, A., Nichol, W., 2009. Management practices for forage brassicas. Ministry of Agriculture and Forestry. Christchurch, New Zealand.

Dillon, P., 2006. Achieving High Dry Matter intake from pasture with grazing dairy cows, in: Elgersma, A., Dijkstra, J., Tamminga, S. (Eds.), Fresh Herbage for Dairy Cattle. Springer. Wageningen, Netherlands, pp. 1-26.

Eckard, R.J., Salardini, A.A., Hannah, M., Franks, D.R. 2001. The yield, quality and irrigation response of summer forage crops suitable for a dairy pasture renovation program in north-western Tasmania. Australian Journal of Experimental Agriculture 41, 37-44.

García S.C., Fulkerson W.J., Brookes S.U., 2008. Dry matter production, nutritive value and efficiency of nutrient utilization of a complementary forage rotation compared to a grass pasture system. Grass and Forage Science 63, 284-300.

Holmes, C.W., Brookes, I.M., Garrick, D.J., MacKenzie, D.D.S., Parkinson, T.J., Wilson, G.F., 2002., Milk Production from Pasture. Massey University. Palmerston North, New Zealand.

Instituto de Investigaciones Agropecuarias (INIA), 2016. Red Agrometeorológica de INIA. http://agromet.inia.cl (acceso, 29.02.2016)

Jacobs, J., Hargreaves, A., 2002. Feeding Dairy Cows. A manual for use in the Target 10 Nutrition Program. $3^{\circ} \mathrm{Ed}$. Department of Natural Resources and Environment Victoria, Australia.

Keim, J.P., Anrique, R., 2011. Nutritional strategies to improve nitrogen use efficiency by grazing dairy cows. Chilean Journal of Agricultural Reserach 71(4), 623-633.

Keim, J.P., López, I.F., Balocchi, O.A., 2015. Sward herbage accumulation and nutritive value as affected by pasture renovation strategy. Grass and Forage Science 70: 283295.

Kolver, E.S., de Veth, M.J., 2002. Prediction of Ruminal pH from Pasture-Based Diets. Journal of Dairy Science 85(5), 1255-1266.

Kolver, E.S., 2003. Nutritional limitations to increased production on pasture-based systems. Proceedings of the Nutrition Society 62, 291-300.

Krause, M., Oetzel, G., 2006. Understanding and preventing subacute ruminal acidosis in dairy herds: a review. Animal Feed Science Technology 126, 215-236.

Lanuza, F., 2011. Suplementos Alimenticios, in: Pulido, R.,
Parga, J., Lanuza, F., Balocchi, O. (Eds.), Suplementación de vacas lecheras a pastoreo. Consorcio Tecnológico de la Leche S.A. Osorno, Chile. pp. 21-48.

Moate, P., Dalley, D., Martin, K., Grainger, C., 1998. Milk production responses to turnips fed to dairy cows in mid lactation. Australian Journal of Experimental Agriculture. 38, 117-123.

Moate, P., Dalley, D., Roche, J., Grainger, C., Hannah, M., Martin, K., 1999. Turnips and protein supplements for lactating dairy cows. Australian Journal of Experimental Agriculture 39, 389-400.

Moate, P.J., Dalley, D.E., Roche, J.R., Gow, C.B., Grainger, C., 2002. Effects on milk production of increased dietary crude protein by feeding nitogen-fertilised turnips or lupins to dairy cows in mid-lactation. Australian Journal of Experimental Agriculture 42(1), 1-6.

Parga, J., Lanuza, F., Pulido, R., Balocchi, O., Canto, F., Campo, S., Uribe, C., 2009. Suplementación estival de vacas lecheras a pastoreo con Nabo forrajero (Brassica rapa L.). Libro de resúmenes XXXIV Congreso de la Sociedad Chilena de Producción Animal, Pucón, Chile. pp. 73-74

Parga, J., Barrientos, L., Pulido, R., Canto, F., Lanuza, F., Balocchi, O., Uribe, C., 2010. Suplementación estival de vacas lecheras a pastoreo con Nabo forrajero (Brassica rapa L.): Respuesta productiva. Libro de resúmenes XXXV Congreso de la Sociedad Chilena de Producción Animal, Coyhaique, Chile. pp. 117-118.

Pulido, R. G., R. Muñoz, C. Jara, O. A. Balocchi, J. P. Smulders, F. Wittwer, P. Orellana, and M. O'Donovan., 2010. The effect of pasture allowance and concentrate supplementation type on milk production performance and dry matter intake of autumn-calving dairy cows in early lactation. Livestock Science 132(1-3), 119-125.

Ruíz-Albarrán, M., Balocchi, O.A., Noro, M., Wittwer, F. and Pulido, R.G., 2012. Effect of increasing pasture allowance and grass silage on animal performance, grazing behaviour and rumen fermentation parameters of dairy cows in early lactation during autumn. Livestock Science 150, 407-413.

Torres, A., 2013. Evaluación y Manejo Agronómico de Cultivos Suplementarios por Macrozonas Homogéneas, in: Oltra, O. (Ed.), Informes Finales Proyectos FIA, Vol. II. Consorcio Tecnológico de la Leche. Osorno, Chile, pp. 253 - 272.

Valentine, I., Kemp, P., 2007. Pasture and supplement resources, in: Rattray, P.V., Brookes, I.M., Nicol, A.M. (Eds.), Pastures and Supplements for Grazing Animals: New Zealand Society of Animal production Occasional Publication No. 14., Hamilton NZ, pp. 3-11.

Westwood C.T., Mulcock H., 2012. Nutritional evaluation of five species of forage brassica. Proceedings of the New Zealand Grassland Association 74: 31-38.

White, J.G.H., Matthew, C., Kemp, P.D., 1999. Supplementary Feeding Systems, in: White, J. and Hodgson, J. (Eds.), Pasture and Crop Science. Oxford University Press. Auckland NZ, pp. 29-44.

Zebeli, Q., Aschenbach, J.R., Tafaj, M., Boguhn, J., Ametaj, B.N., Drochner, W., 2012. Invited review: Role of physically effective fiber and estimation of dietary fiber adequacy in high-producing dairy cattle. Journal of Dairy Science 95(3), 1041-1056. 